\title{
Neutrino hierarchy and fermion spectrum from a single family in six dimensions: realistic predictions
}

\author{
J.-M. Frère, ${ }^{a}$ M. Libanov, ${ }^{b, c}$ S. Mollet ${ }^{a}$ and S. Troitsky ${ }^{b}$ \\ ${ }^{a}$ Service de Physique Théorique, Université Libre de Bruxelles, \\ Campus de la Plaine CP225, Bd du Triomphe, 1050 Brussels, Belgium \\ ${ }^{b}$ Institute for Nuclear Research of the Russian Academy of Sciences, \\ 60th October Anniversary Prospect 7a, 117312, Moscow, Russia \\ ${ }^{c}$ Moscow Institute of Physics and Technology, \\ Institutskii per., 9, 141700, Dolgoprudny, Moscow Region, Russia \\ E-mail: frere@ulb.ac.be, ml@ms2.inr.ac.ru, smollet@ulb.ac.be, \\ st@ms2.inr.ac.ru
}

\begin{abstract}
In this paper, we move from a "proof of concept" to challenging predictions in a flavour model emerging from a single fermion family in six dimensions (6-D) with the two extra dimensions compactified on a sphere. The most striking predictions (beyond the basic ingredients already demonstrated for a realistic quark mass pattern) are for the neutrino sector. Not only can 4-D Majorana masses be generated from 6-D (where no Majorana spinors exist), but Majorana masses are naturally associated to large mixing. The model favours inverted hierarchy and a quasi-Dirac partial suppression of neutrinoless double beta decay.
\end{abstract}

KEYwords: Large Extra Dimensions, Quark Masses and SM Parameters, Neutrino Physics 


\section{Contents}

1 Introduction 1

2 The model: a general description 3

3 Scalar parameters $\quad 4$

3.1 Scalar profiles 4

$\begin{array}{ll}3.2 & \text { The } X \text { field and the pseudo-Goldstone mode }\end{array}$

$\begin{array}{lll}3.3 & \text { Fitting the scalar-boson mass } & 7\end{array}$

4 Fermion parameters $\quad 7$

4.1 Selection rules and hierarchies for charged fermions and neutrinos $\quad 7$

$\begin{array}{lll}4.2 & \text { Fitting the masses and mixings } & 10\end{array}$

$\begin{array}{llr}5 & \text { Experimental tests } & 11\end{array}$

6 Conclusions 14

\section{Introduction}

In the recent couple of years, the particle physics was faced with two important experimental discoveries. One is the new boson with mass about $125 \mathrm{GeV}[1,2]$ whose properties look now very similar $[3,4]$ to those of the Standard Model Scalar boson required by the BroutEnglert-Higgs mechanism $[5,6]$. The other one is the nonzero mixing between the first and the third neutrino generations whose value was determined in several experiments, with growing precision $[7,8]$. While the first one represents a triumphal support to the Standard Model (SM), the second one reminds us how little we understand the flavour sector. Neutrino mixing is impossible in the SM where neutrinos are strictly massless. While neutrino masses can in principle be added to the SM with the help of right-handed neutrinos and very small Yukawa couplings, the alternate possibility (Majorana or Weyl neutrinos) is quite attractive. However, we still do not have a clue to the physical realization of this mechanism.

One of the approaches, which allows to explain naturally the hierarchy of masses and mixings both in the quark and lepton sectors but does not necessarily require new physics at the LHC scale, explores the idea of extra space dimensions with the SM matter localized on a brane or a topological defect. We have shown in previous papers how the topological structure can be chosen in a 6-D model for existence of three effective four-dimensional modes for each multidimensional fermion. (Three is for the moment an arbitrary - empirically motivated - choice; we will however argue that four families would be difficult to fit within the experimental constraints). Thus a single generation of 
fermions exists in the fundamental theory, with a corresponding reduction of the number of free parameters. Meanwhile, the correct pattern of masses and mixings is generated without fine tuning by overlaps of the wave functions. This rigidity of the framework is strikingly different from "multilocalisation" schemes, where various fermions are located on branes with tuned position in the 5th dimension. A working example with six space-time dimensions and the Abelian vortex as a brane has been constructed [9, 10] and elaborated [11-18]. We have later shown that the very same 6-D mechanism, can provide 4-D Majorana (Weyl) neutrinos, an astonishing fact in itself, since Majorana fermions don't exist in 6-D. This surprising possibility comes with tantalizing implications: it implies naturally large mixings and an inverted hierarchy for masses in the neutrino sector [19] (see refs. [20, 21]).

In this note, we go beyond the "proof of concept", further discuss the latest developments in our six-dimensional approach to the flavour structure of the SM, but also provide a convincing fit to the mass and mixing spectra. Beyond this specific fit, we also outline what we consider to be the "generic" features of the model, and suggest possible tests. We also consider briefly (in section 5) the possibility to study these key features in an ad-hoc effective 4-D approach, in particular for test of flavour-changing but family-number conserving interactions. This allows to put purely experiment-based limits for possible detection of flavour-carrying new particles at LHC.

While we had already stressed some of these points (notably the need for a light Standard Model Scalar $[17,18]$ ), the latest data allow to narrow the fit. In terms of quarks, we reproduce the mixing structure with a minimal number of parameters, and obtain decent values for the masses. In terms of neutrinos, large mixing (for Majorana neutrinos in four dimensions) appear naturally [19]. While we already announced (in tempore non suspecto) a finite value of $\theta_{13}$ (now seen to be compatible with experiment), we confirm the distinctive prediction of inverted hierarchy and nearly pseudo-Dirac mass matrix, which minimizes the neutrinoless double beta decay (that is, its value is found at the very bottom of the inverted hierarchy allowed band). CP violation in neutrino oscillations is also expected.

One major characteristic is the presence of some family-number symmetry. In the absence of CKM this is indeed exact, and leads to conservation of "family number". We have shown previously that this "horizontal symmetry", mediated by Kaluza-Klein (KK) excitations, is perfectly compatible with existing data, but puts a lower bound on the 6-D scale. It provides however for interesting phenomenology, and may be a way of testing the model. Note that these "horizontal" interactions are not directly responsible for the mass generation, and thus avoid the major conflict with experiment seen, for instance, in many "extended technicolor" models. The $\sim 50 \mathrm{TeV}$ lower bound (obtained from limits on flavour-changing processes like $K_{L}^{0} \rightarrow \bar{e} \mu$ ) results from the estimations of wave functions overlaps in the specific case considered (compactification on a sphere). It is also possible to write the interaction in a way independent of these overlaps, in an effective approach. In that case, possible discovery at LHC can be related to the precision tests in a modelindependent way.

The paper is organized as follows. We briefly review the construction of the model in section 2. Then, we demonstrate explicitly how the observed value of the Standard Model Scalar mass fits the overall construction and fix the value of parameters in the scalar 
sector (section 3). A possible Goldstone mode is avoided through the Yukawa couplings. We present the fermionic interactions and fit masses and mixing of the matter fields in section 4. This successful simultaneous fit of parameters related to scalar, charged-fermion and neutrino sectors allows us to proceed with an update on quantitative experimental predictions, distinctive for our model (and a possible model-independent approach), in section 5 , while we conclude briefly in section 6 , and mention some possible alternatives to the choices made in this paper.

\section{The model: a general description}

The basic model has been presented in successive forms, first [9] with a minimal number of fields in flat space. Successive versions introduced a more detailed structure of scalar fields, which we outline here, and later brought the compactification to a sphere (which avoids the problem of gauge bosons localization). This choice of compactification is clearly not unique, though representative. In particular, warped compactifications, like those considered in ref. [22], lead to a similar pattern of masses and mixings.

The basic set-up consists of a vortex structure (which acts as a kind of background). It is created by a scalar field $\Phi$, charged under a gauged $U(1)_{\mathrm{g}}$, to which is associated the gauge field ${ }^{1} A^{M}$. Fermions are introduced as one 6-D fermion field for each charge of fermions (that means one $U$ field, which will later generate the right-handed (4-D) up, charmed and top quarks, and so on for $D$ down-type quark, $Q$ quark doublet, $E$ lepton singlet and $L$ lepton doublet field). A singlet fermion $N$ is also included. Here we have a choice: if we wish to deal with 4-D Dirac neutrinos, light right-handed neutrinos are needed, and $N$ must couple to the vortex structure. While this choice is perfectly possible, we take here the alternate view, and seek a 4-D Majorana solution for the physical neutrinos. The $N$ field will remain a "bulk" field in this paper, but will provide the Majorana masses. These fermion fields are seen as 8-component fermions in 6-D, but their couplings can be distinguished in terms of 6-D "chirality" - to avoid later confusion we will call it $\Gamma_{7}$ parity, and the $A^{M}$ fields couples unequally to the "+" and "-" $\Gamma_{7}$ parity components of the fermion fields.

A scalar doublet field $H$ provides the usual Brout-Englert-Higgs (BEH) electroweak symmetry breaking, and also will contribute to the fermion masses. More fields are needed to generate realistic masses (in particular, to generate the off-diagonal couplings leading to mixing), and we could choose to introduce them as needed (for instance, an extra scalar doublet). Since renormalizability is anyway lost in 6 - $\mathrm{D}$, we choose instead to minimize the number of degrees of freedom by factorizing the interaction. Thus, a single $S U(2) \times U(1)$ singlet, $X$ is introduced. In the same way, since we will need a winding-3 vortex, we could request the $\Phi$ field to exhibit such a solution, but in practice, we prefer to keep $\Phi$ winding to 1 , and introduce a $\Phi^{3}$ couplings to the fermions. Of course, the opposite option could be taken, at the cost of multiplying the fields.

\footnotetext{
${ }^{1}$ Our notations coincide with those used in Refs. [9, 14]. In particular, 6-D coordinates are labeled by capital Latin indices $A, B=0, \ldots, 5$. Four-dimensional coordinates are labeled by Greek indices, $\mu, \nu=$ $0, \ldots, 3$. The signature is mostly negative.
} 
Even with this restricted set of fields, various assignations of the $U(1)_{\mathrm{g}}$ vortex charge are possible, and depend in particular on the choice of flat or spherical geometry. Therefore, we are dealing with a class of models, of which we select the minimal one for further quantitative development. The present model is determined by the charge assignments of the fields listed in table 1 . We note that the choice of charges in the scalar sector is slightly

\begin{tabular}{|c|c|c|c|c|c|}
\hline \hline Field & Notation & $U(1)_{g}$ & $U(1)_{Y}$ & $S U(2)_{w}$ & $S U(3)_{c}$ \\
\hline \hline vortex scalar & $\Phi$ & +1 & 0 & $\mathbf{1}$ & $\mathbf{1}$ \\
\hline BEH boson & $H$ & 0 & $+1 / 2$ & $\mathbf{2}$ & $\mathbf{1}$ \\
\hline auxiliary scalar & $X$ & +1 & 0 & $\mathbf{1}$ & $\mathbf{1}$ \\
\hline \hline quark $S U(2)_{\mathrm{W}}$ doublet & $\left(Q_{+}, Q_{-}\right)$ & $(3,0)$ & $1 / 6$ & $\mathbf{2}$ & $\mathbf{3}$ \\
\hline up-type quark $S U(2)_{\mathrm{W}}$ singlet & $\left(U_{+}, U_{-}\right)$ & $(0,3)$ & $2 / 3$ & $\mathbf{1}$ & $\mathbf{3}$ \\
\hline down-type quark $S U(2)_{\mathrm{W}}$ singlet & $\left(D_{+}, D_{-}\right)$ & $(0,3)$ & $-1 / 3$ & $\mathbf{1}$ & $\mathbf{3}$ \\
\hline lepton $S U(2)_{\mathrm{W}}$ doublet & $\left(L_{+}, L_{-}\right)$ & $(3,0)$ & $-1 / 2$ & $\mathbf{2}$ & $\mathbf{1}$ \\
\hline charged lepton $S U(2)_{\mathrm{W}}$ singlet & $\left(E_{+}, E_{-}\right)$ & $(0,3)$ & -1 & $\mathbf{1}$ & $\mathbf{1}$ \\
\hline sterile neutrino singlet & $\left(N_{+}, N_{-}\right)$ & $(0,0)$ & 0 & $\mathbf{1}$ & $\mathbf{1}$ \\
\hline \hline
\end{tabular}

Table 1. Charge assignments of the fields under the gauge groups of the SM and under $U(1)_{\mathrm{g}}$. For fermions, the two numbers in parentheses correspond to the charges of the components with positive and negative values of the $\Gamma_{7}$ parity, denoted everywhere by "+" and "-" indices, correspondingly.

different with respect to our previous work. The possibility of such variants is discussed in section 6 .

\section{$3 \quad$ Scalar parameters}

\subsection{Scalar profiles}

In a first step, we adjust (within the above charge assignment) the model parameters to generate the desired scale for electroweak symmetry breaking (we anticipate here somewhat to the fact the KK scale will be bounded from below by the absence of flavour-changing neutral currents). This involves fixing the potential and couplings of $\Phi, H$ (and also the extra field $X$ ). The two extra dimensions are compactified on a sphere of radius $R$, with the spherical coordinates $\theta, \phi$.

Let us suspend for the moment the field $X$ which will be required to generate fermion mixings. We will switch it on back in the next subsection. As announced, the BEH field $H$ acquires a non-constant profile in transverse dimensions due to its interaction with the scalar $\Phi$. The role of the $H$ field in the model requires it to transform under the SM electroweak gauge group $S U(2)_{\mathrm{W}} \times U(1)_{\mathrm{Y}}$ as usual. In the $\mathrm{SM}$, the electroweak symmetry is broken by the BEH-field vacuum expectation value provided by the potential term $\sim\left(|H|^{2}-\mu^{2}\right)^{2}$. In our case, the non-constant $H$ profile is obtained with the help of 
the coupling between $\Phi$ and $H$, so the complete Lagrangian for them reads [17, 18]:

$$
\mathcal{L}_{\Phi+H}=R^{2} \sin \theta\left(\left|D_{A} \Phi\right|^{2}+\left|D_{A} H\right|^{2}-\frac{\lambda}{2}\left(|\Phi|^{2}-v^{2}\right)^{2}-\frac{\kappa}{2}\left(|H|^{2}-\mu^{2}\right)^{2}-h^{2}|H|^{2}|\Phi|^{2}\right) .
$$

Consider the last three terms of this expression. The coupling $h^{2}|H|^{2}|\Phi|^{2}$ enforces "orthogonalization" of the two scalar fields in the sense that the larger value of one corresponds to a reduced value of the other. The topology of the vortex determines the global profile in which $H(\theta)$ goes to zero at sufficiently large $\theta$. At the origin, where $\Phi \rightarrow 0$, the last term loses its importance while the usual $\sim\left(|H|^{2}-\mu^{2}\right)^{2}$ term enforces a nonzero value of $H$. For sufficiently large $h^{2}$, this value gets smaller and the entire solution approaches the trivial one which is phenomenologically inappropriate. In the opposite case of very small $h^{2}$, the solution for $H^{2}$ approaches its vacuum value $\mu^{2}$. This construction is analogous to that of Witten [23] where a similar problem has been solved in four dimensions.

To find a nonsingular solution, one imposes the following boundary conditions,

$$
H^{\prime}(0)=0, \quad H(\pi)=0 .
$$

These conditions agree with the physical picture suggested above. Note that the condition $H^{\prime}(0)=0$ does not fix the $H$ value at the origin: instead, this value is determined by parameters of the potential. The latter are constrained by the requirement that the solution should reproduce the proper SM BEH vev [15],

$$
\frac{V_{S M}^{2}}{2}=2 \pi R^{2} \int_{0}^{\pi} \mathrm{d} \theta \sin \theta H^{2}(\theta)
$$

where $V_{S M} \approx 246 \mathrm{GeV}$ fixes the energy scale of the electroweak symmetry breaking and determines, e.g., the W-boson mass.

The orthogonalization effect ensures that the characteristic extension $\theta_{H}$ of the profile of the field $H$ is of the same order as $\theta_{\Phi}$, the characteristic extension of $\Phi$. In practice, we always have $\theta_{H} \lesssim \theta_{\Phi} \ll 1$ which allows to simplify the normalization integral,

$$
\frac{V_{S M}^{2}}{2} \approx \pi R^{2} \theta_{\Phi}^{2} H^{2}(0)
$$

where we approximated $\sin \theta \approx \theta$, put $H(\theta) \approx H(0)$ on the interval $\left[0, \theta_{\Phi}\right]$ and $H(\theta) \approx 0$ outside it. We further define

$$
\Lambda_{\mathrm{V}} \equiv 1 /\left(R \theta_{\Phi}\right)
$$

a parameter with the dimension of energy which sets up the vortex scale. We obtain

$$
H(0) \approx\left(\frac{0.1 \mathrm{TeV}}{\Lambda_{\mathrm{V}}}\right) \Lambda_{\mathrm{V}}^{2}
$$

As we will see below (section 5), phenomenological constraints from the absence of rare processes require $\Lambda_{\mathrm{V}} \sim 10^{4} \mathrm{TeV}$, which implies $H(0) \approx 10^{-5} \Lambda_{\mathrm{V}}^{2}$. One may note that this fine tuning is a weak point of the model; however, it is a reflection of the unexplained adjustment of parameters required to obtain the electroweak scale in the SM. It is tempting 
to argue for a 6-D dynamical reason for this tuning, cf. [24, 25]. We stress that this is the only critical fine-tuning in the model (as in grand unified theories).

By a careful study of the solution for the scalar fields, together with the normalization requirement, eq. (3.2), one may obtain an interesting approximate relation, whose technical derivation is beyond the scope of the present work,

$$
m_{\mathrm{H}} \simeq \kappa \mu \times 70 \mathrm{GeV}
$$

for the 4-D mass of the observable scalar-boson mode. One may note that the dimensionless combination $\kappa \mu$ plays the role of a coupling constant in the 6 -D model and therefore should not be too large, say $\kappa \mu \lesssim 4 \pi$, to keep perturbativity. Therefore, weak coupling implies a relatively light $\mathrm{BEH}$ boson in this model $[17,18]$. It is interesting to note that this single tuning of the 6-dimensional solution results in effectively decoupling the EW breaking sector from the $1 / R$ scale, which makes it almost indistinguishable from a pure 4 -D version of the SM.

The numerical search for the scalar solution is technically very difficult because the trivial solution is very close to the interesting one, so the standard procedure often finds the trivial, though unstable, solution. Theoretical considerations have suggested [17, 18], however, a way to find the stable solution which will be used below.

\subsection{The $X$ field and the pseudo-Goldstone mode}

As explained in our previous works $[9,10]$, the model with two scalar fields, $\Phi$ and $H$, possesses an exact symmetry, the family number, which is related to the geometry of the extra dimensions and counts the winding number of the six-dimensional fields. While the proper hierarchical masses of quarks and charged leptons may be generated in this way, the CKM mixings are forbidden by this symmetry. This is achieved by introducing the field $X$, cf. table 1 . The bosonic Lagrangian should be supplemented with

$$
\mathcal{L}_{X}=R^{2} \sin \theta\left(\left|D_{A} X\right|^{2}-\frac{\rho}{2}\left(|X|^{2}-v^{2}\right)^{2}-\eta^{2}|X|^{2}|\Phi|^{2}\right) .
$$

In our basic formulation the scalar potential for $X$ and $\Phi$ is insensitive to their relative phase, and thus possesses two global $U(1)$ symmetries which correspond to independent phase rotations of $X$ and $\Phi$. Both symmetries are broken by nonzero scalar profiles we require. This leads to two Goldstone bosons of which one combination is absorbed by $A^{M}$ to give it mass while the other, which we will call $Y$ is left massless at this stage.

Would such a (pseudo-) Goldstone boson be phenomenologically excluded? Its couplings to light particles are determined by the Lagrangian, and depend upon the evaluation of the corresponding overlaps; in general they are suppressed by a factor of order $\langle H\rangle /\langle X\rangle$ with respect to the Standard Model Scalar. The charge assignment we have chosen, table 1, guarantees that these couplings are furthermore off-diagonal in flavour (the key point here is the charge of $H$ under $U(1)_{\mathrm{g}}$ which we selected in this paper expressly for this reason). However, even such reduced couplings, which effectively suppress virtual processes are dangerous if the pseudo-goldstone particle is (nearly) massless, a typical test being $\mu \rightarrow e Y$ decay. 
In fact, we may notice that there are no anomalies associated to the $Y$ current, hence no axion-like behaviour, but on the other hand, the full Lagrangian violates the associated symmetry at the level of the Yukawa couplings, see table 3. Mass contributions (determined by the high scale and the Yukawa couplings) would thus be generated at loop level, which is enough to exclude production of $Y$ in low-energy processes. Its coupling suppression then brings the expected limits in a line with those from KK gauge-mediated flavour-changing currents. Note in passing that there is no "lowest stable KK mode" in this model, which could serve as a dark matter candidate. As in the Standard Model, dark matter should be added explicitly, but is not considered in the present publication.

We could of course introduce $a b$ initio a coupling between the phases of $X$ and $\Phi$, like the one induced by these loop effects; the difficulty then is in resolving coupled differential equations in the 2-D space; we have preferred to keep this mass generation as a later perturbation of the "scalar background" due to the fermionic fields.

\section{3 $\quad$ Fitting the scalar-boson mass}

The approach to numerical fitting of the scalar parameters, quite nontrivial by itself, was outlined in ref. $[17,18]$. One should take care of two principal constraints whose origin lays outside of the scalar sector. One is the relation between characteristic extensions $\theta_{\Phi}$ and $\theta_{A}$ of the, respectively, scalar and gauge fields forming the vortex,

$$
\sigma=\theta_{\Phi} / \theta_{A} \ll 1
$$

which ensures [14] that the fermions may acquire hierarchical masses, and another is

$$
1 / R \gtrsim 50 \mathrm{TeV}
$$

as required by the absence of dangerous flavour-changing processes, see ref. [15] and section 5. In practice, the field $X$ is treated as a perturbation so the first step is to obtain the correct scalar mass and effective vacuum expectation value, eq. (3.2), by solving the problem for $A, \Phi$ and $H$. Skipping details, we obtain the parameters of the bosonic Lagrangian listed in table 2. With the overall scale $\Lambda_{\mathrm{V}} \simeq 7700 \mathrm{TeV}$ we reproduce both parameters of the Standard Model scalar sector, $m_{H} \simeq 125 \mathrm{GeV}$ and $V_{S M} \simeq 246 \mathrm{GeV}$. We note here that the number of parameters in the scalar sector of our model exceeds that of the SM; this will be compensated by a highly predictive fermionic sector which we consider in the next section.

\section{Fermion parameters}

\subsection{Selection rules and hierarchies for charged fermions and neutrinos}

The topology of the background bosonic field provides a certain number of fermionic chiral zero modes thanks to the index theorem, as explained e.g. in [26] for $(2+1)$ dimensions and in [23] for $(3+1)$ dimensions. In our case, the coupling is selected to have the topological number three, so three zero modes appear in the effective $(3+1)$-dimensional theory for each $(5+1)$-dimensional fermion. These modes are linearly independent which in our case 


\begin{tabular}{|c|l|}
\hline \hline$R$ & $77.0 \Lambda_{\mathrm{V}}^{-1}$ \\
\hline$e$ & $0.01 \Lambda_{\mathrm{V}}^{-1}$ \\
\hline$v$ & $1.00 \Lambda_{\mathrm{V}}^{2}$ \\
\hline$\lambda$ & $1.00 \Lambda_{\mathrm{V}}^{-2}$ \\
\hline$\mu$ & $0.50 \Lambda_{\mathrm{V}}^{2}$ \\
\hline$\kappa$ & $4.22 \Lambda_{\mathrm{V}}^{-2}$ \\
\hline$h^{2}$ & $1.64 \Lambda_{\mathrm{V}}^{-2}$ \\
\hline$v$ & $1.50 \Lambda_{\mathrm{V}}^{2}$ \\
\hline$\rho$ & $0.50 \Lambda_{\mathrm{V}}^{-2}$ \\
\hline$\eta^{2}$ & $1.20 \Lambda_{\mathrm{V}}^{-2}$ \\
\hline
\end{tabular}

Table 2. Parameters of the compactification and of the scalar sector. We have rounded up the value of $\kappa$ which is tuned to a higher precision, as discussed in the text, to ensure the low value of the weak scale compared to the vortex size.

guarantees that they have different winding numbers, $\mathrm{e}^{i k \phi}$ with $k=0,1,2$, in the two extra dimensions. The different windings imply in turn the different behaviour, $\sim \theta^{k}$, at the origin. Upon the electroweak symmetry breaking, that is in the presence of $H \neq 0$, these zero modes are lifted and the hierarchical mass matrix emerges [9, 10, 14], with the values determined by the overlaps between the scalar and fermion profiles, multiplied by the Yukawa couplings. Our model includes the following coupling of fermions to the vortex:

$$
\begin{aligned}
& g^{\Psi} \Phi^{k} \bar{\Psi}_{+} \Psi_{-}+\text {h.c. } \quad \text { will provide the left-handed 4-D species, } \\
& g^{\Psi} \Phi^{k} \bar{\Psi}_{-} \Psi_{+}+\text {h.c. } \quad \text { will provide the right-handed 4-D species, }
\end{aligned}
$$

where $\Psi=\left(\Psi_{+}, \Psi_{-}\right)$stands for $Q, U, D, L$ and $E$ 6-D fermions and $k$ gives the number of 4 -D zero modes for each of these species. The charges for fermions $\left(Q\left(\Psi_{+}\right), Q\left(\Psi_{-}\right)\right)$under the vortex $U(1)_{g}$ group must be chosen such that $\left|Q\left(\Psi_{+}\right)-Q\left(\Psi_{-}\right)\right|=k Q(\Phi)$. In our working example, $k=3$ and the assignments are given in the third column of table 1. It's worth mentioning that the 4-D chirality is determined by the sign of $Q\left(\Psi_{+}\right)-Q\left(\Psi_{-}\right)$. The zero modes are chiral also in the six-dimensional sense, which means that in a particular ("chiral") representation of 6-D Dirac matrices, see [9], certain components of 8-component spinors are zero. In particular, for the quarks we have the following structure of the zero modes,

$$
Q_{k}=\left(\begin{array}{c}
0 \\
\mathrm{e}^{-i \phi(k-3)} \ldots \\
\mathrm{e}^{-i \phi(k-1)} \ldots \\
0
\end{array}\right), \quad U_{k}=\left(\begin{array}{c}
\mathrm{e}^{-i \phi(k-1)} \ldots \\
0 \\
0 \\
\mathrm{e}^{-i \phi(k-3)} \ldots
\end{array}\right)
$$

$k=1,2,3$, and similarly for the $S U(2)_{\mathrm{W}}$-singlet (right-handed) $D$ quarks (in 6-D, spinors are 8-component and each element here represents a bispinor). The couplings which lift the quark zero modes read as

$$
Y_{d} H \bar{Q}_{+} D_{-}+Y_{u} \tilde{H} \bar{Q}_{+} U_{-}
$$


where $\tilde{H}$ is obtained from $H$ by the action of the antisymmetric tensor $\epsilon$ in the $S U(2)$ space, $\tilde{H}_{i}=\epsilon_{i j} H_{j}$. These interaction terms result in the effective four-dimensional mass matrix

$$
m_{k l}=\int d \phi d \theta H \bar{Q}_{k} D_{l}
$$

and the structure (4.1) results in,

$$
m_{k l} \propto \delta_{k l} \cdot \sigma^{2(3-k)},
$$

where the selection rule $m_{k l} \sim \delta_{k l}$ comes from the $\phi$ integration, while the $\theta$ integration gives rise to hierarchical dependence on $\sigma$. The selection rule is of course a manifestation of the family-number symmetry; it leads to a diagonal but (because of overlaps in $\theta$ integration) strongly hierarchical mass matrix. The off-diagonal elements, corresponding to the CKM mixing, can be populated by introducing an additional field $X$ which allows to write interactions with $\Phi$ whose profile breaks the 6-D rotational symmetry, and therefore the family-number symmetry, spontaneously. The hierarchy of the mass matrix elements is generated by a small parameter $\sigma=\theta_{\Phi} / \theta_{A}$.

For charged leptons, the interaction which produces diagonal masses is given by the similar term

$$
Y_{e} H \bar{L}_{+} E_{-}
$$

which results in a similar hierarchical mass structure due to similar wave-function profiles. It would be also the case for neutrinos if they had a Dirac mass (see [11]); however, for the effective 4-D Majorana masses, the situation is quite different [19]. The corresponding interaction is given by

$$
Y_{n} \tilde{H} \bar{L}_{-} N_{+}
$$

for the $N$ field. In the present approach (where we seek light Majorana neutrinos in 4-D), the $N$ field remains a "bulk" field (no light modes), and does not have coupling to the vortex. The wave functions of its modes should be normalized and their absolute value is therefore suppressed, thus diminishing the overlaps. There exists a 6 -D mass term for the $N$ field,

$$
\frac{M}{2} \bar{N}^{c} N+\text { h.c. }
$$

Of course, no Majorana fermions exist in 6-D. The above term (eq. (4.3)), while evocative of a Majorana mass term, in fact couples $N_{+}$to $N_{-}$. Still leptonic number violation results from the Yukawa couplings, and eq. (4.3) leads to light 4-D Majorana masses suppressed by the see-saw mechanism for the physical neutrinos [19]. While the zero modes of the field $L$ have a Lorentz structure similar to eq. (4.1), the structure of the Majorana interaction results in a different selection rule for the masses,

$$
m_{k l} \propto \delta_{4, k+l} \cdot \sigma^{3-|k-l| / 2},
$$

which therefore provides a very different (anti-diagonal) hierarchical structure, which is in the end surprisingly similar to that which emerges from the experimental data. The overall suppression of neutrino masses is achieved by the usual see-saw mechanism, where the large 
mass for the singlet field $N$, which is not localized on the brane, appears as a parameter of the model which is naturally of order of the compactification scale. This structure is the direct cause for the most striking feature of the model, large lepton mixing [19].

It is important to note that $\sigma$ in eq. (4.4) is the same as in eq. (4.2), so that both hierarchies are governed by the same parameter. It determines also the value of the neutrino mixing angle $\theta_{13} \sim \sigma$.

\subsection{Fitting the masses and mixings}

Therefore, the mechanism we advocate incorporates two very different hierarchies within a single framework. However, the hierarchies of masses of charged fermions differ (e.g. for the quarks, the mass ratios are much more severe for the "up" sector than for the "down" sector). Various strategies are possible to get realistic masses and mixing. This can be fixed, either through mixing terms, or by choosing the initial parameters to optimize the diagonal ratios, hence different profiles for the $U$ and $D$ fermions. We present here a benchmark choice of parameters which allow for a nice fit of the experimental masses and mixings.

Since the fermion masses experience renormalization-group running (especially important for strongly-interacting quarks), at which energy scale should we fit the masses ${ }^{2}$ ? Clearly, it is hard to give a precise answer. In our mechanism, like in the SM, fermion masses are associated with the electroweak breaking, so it is natural to calculate their values at this scale. We therefore use the values at $M_{Z}$, the scale which is commonly used. One shall note however that for light quarks, the mass running in the strong-coupling regime (momenta $\lesssim \Lambda_{\mathrm{QCD}}$ ) is uncertain. As a result, in our fit, we concentrate on obtaining more precise values for masses of heavier quarks and all leptons, as well as for mixing angles (whose running is negligible [27]), relaxing to some extent uncertainties in masses of $u$ and $d$ quarks.

Parameters of the model in this particular fit are listed in table 3. The values of the mass-matrix observables for this fit, together with their experimental values [28-30] (calculated at $M_{Z}$ when applicable), are given in table 4 .

Variations of the model, namely relaxing the quark-lepton universality of $U(1)_{g}$ charges (which we prefer to avoid), may allow for a slightly better fit.

Note that in the SM, redefinition of fields may always be used to remove all unphysical phases from the mass matrix, so that only one physical $C P$-violating phase remains in CKM. It is also true here; however, we restricted ourselves for simplicity (in a first approximation) to real couplings and succeeded in obtaining a reasonable set of quark masses and mixing angles. We clearly could not (and did not) obtain the $C P$-violating phase in this way. We can introduce it by means of a free complex phase parameter in the couplings but we cannot predict it. This problem will be studied elsewhere, with ref. [32] providing one of possible frameworks.

The opposite happens in the leptonic sector. Here, we needed to use complex couplings to obtain a reasonable fit to the experimental masses and mixings. Therefore, the model

\footnotetext{
${ }^{2}$ The first one who asked this question was M. Voloshin.
} 


\begin{tabular}{|l|l|}
\hline \hline \multicolumn{2}{|c|}{ Fermion-vortex couplings } \\
\hline$g_{L}^{Q}$ & $0.020 \quad \Lambda_{\mathrm{V}}^{-5}$ \\
\hline$g_{R}^{U}$ & $0.005 \Lambda_{\mathrm{V}}^{-5}$ \\
\hline$g_{R}^{D}$ & $0.063 \quad \Lambda_{\mathrm{V}}^{-5}$ \\
\hline$g_{L}^{L}$ & $0.013 \quad \Lambda_{\mathrm{V}}^{-5}$ \\
\hline$g_{R}^{E}$ & $0.013 \quad \Lambda_{\mathrm{V}}^{-5}$ \\
\hline \hline \multicolumn{2}{|c|}{ "Yukawa" fermion-scalars couplings } \\
\hline$H \bar{Q}_{+} D_{-}$ & $7.00 \Lambda_{\mathrm{V}}^{-1}$ \\
\hline$H X^{*} \Phi \bar{Q}_{+} D_{-}$ & $14.0 \Lambda_{\mathrm{V}}^{-5}$ \\
\hline \hline$\tilde{H} \bar{Q}_{+} U_{-}$ & $850 \Lambda_{\mathrm{V}}^{-1}$ \\
\hline$\tilde{H} X^{*} \Phi \bar{Q}_{+} U_{-}$ & $255 \Lambda_{\mathrm{V}}^{-5}$ \\
\hline \hline$H X^{*} \Phi \bar{L}_{+} E_{-}$ & $56 \Lambda_{\mathrm{V}}^{-5}$ \\
\hline$H X \Phi^{*} \bar{L}_{+} E_{-}$ & $-160 \Lambda_{\mathrm{V}}^{-5}$ \\
\hline \hline$\tilde{H} \bar{L}_{-} N_{+}$ & $0.165 \Lambda_{\mathrm{V}}^{-1}$ \\
\hline$\tilde{H} X \Phi^{*} \bar{L}_{-} N_{+}$ & $(0.91+1.04 i) \cdot 10^{-2} \Lambda_{\mathrm{V}}^{-5}$ \\
\hline$\tilde{H}\left(X^{*}\right)^{4} \Phi \bar{L}_{+} N_{-}$ & $1.00 \Lambda_{\mathrm{V}}^{-11}$ \\
\hline \hline
\end{tabular}

Table 3. Benchmark parameters for the 6-D fermionic sector, given in terms of the overall scale $\Lambda_{\mathrm{V}}$ which in our case is $\approx 7700 \mathrm{TeV}$. It is worth noting that, while necessary for mass generation, the last coupling is fully arbitrary and can always be put equal to 1 by redefinition of other Yukawa's couplings in the neutrino sector. Then it can be disregarded in the counting of free parameters of the model.

presented here predicts non zero $C P$ violation in the neutrino sector whose measurable value may be parametrized by the Jarlskog invariant (adapted for leptons), $J$, which we also list in table 4 . Unlike for quarks, $C P$ violation in neutrinos is a prediction of our model, at least in the present fit.

\section{$5 \quad$ Experimental tests}

While the most impressive implications of the 6-D model is in the very different nature of the neutrino sector, it is important to look for other possible signals and tests. We have in previous papers suggested experimental tests and constraints. Here, we give a brief review and update on them.

The most constraining ones are related to the KK excitations of gauge bosons, which carry "winding", i.e. family number. We typically get neutral flavour-changing, but family number-conserving interactions, and a strong bound on $(\varkappa R)^{2}$ is obtained, where $R$ is the compactification scale, and $\varkappa$ is a parameter describing the convolution of the overlap between wave functions of fermions from different families and the gauge-boson KK-mode profile. This suppresses the coupling with respect to the canonical (diagonal) gauge couplings. In previous work $[15,33]$, we have obtained modest suppression factors $\varkappa$, leading to an expected mass scale for the KK excitations, well beyond LHC reach, but still a challenge for increasing precision low energy tests. While the previous calculations were based 


\begin{tabular}{|c|c|c|}
\hline Parameter & Fitted value & Experimental value \\
\hline \multicolumn{3}{|r|}{ The scalar-boson mass } \\
\hline$m_{H}$ & $125 \mathrm{GeV}$ & $\begin{array}{l}125.5 \pm 0.2 \text { (stat.) } \pm 0.6 \text { (syst.) }[3] \\
125.7 \pm 0.3 \text { (stat.) } \pm 0.3 \text { (syst.) }[4]\end{array}$ \\
\hline \multicolumn{3}{|r|}{ Quark masses at Z scale } \\
\hline $\begin{array}{l}m_{d} \\
m_{s} \\
m_{b} \\
m_{u} \\
m_{c} \\
m_{t}\end{array}$ & $\begin{array}{l}0.01 \mathrm{GeV} \\
0.051 \mathrm{GeV} \\
2.86 \mathrm{GeV} \\
0.023 \mathrm{GeV} \\
0.72 \mathrm{GeV} \\
172 \mathrm{GeV}\end{array}$ & $\begin{array}{c}(0.00282 \pm 0.00048) \mathrm{GeV} \\
\left(0.057_{-0.012}^{+0.018}\right) \mathrm{GeV} \\
2.86_{-0.06}^{+0.16} \mathrm{GeV} \\
0.00138_{-0.00041}^{+0.00042} \mathrm{GeV} \\
0.638_{-0.084}^{+0.043} \mathrm{GeV} \\
172.1 \pm 1.2 \mathrm{GeV}\end{array}$ \\
\hline \multicolumn{3}{|r|}{ Quark mixing matrix } \\
\hline$\left|U_{\mathrm{CKM}}\right|$ & $\left(\begin{array}{ccc}0.979 & 0.207 & 0.0015 \\
0.206 & 0.9730 & 0.046 \\
0.011 & 0.049 & 0.999\end{array}\right)$ & $\left(\begin{array}{ccc}0.97427 \pm 0.00015 & 0.22534 \pm 0.00065 & 0.00351_{-0.00014}^{+0.00015} \\
0.22520 \pm 0.00065 & 0.97344 \pm 0.00016 & 0.0412_{-0.0011}^{+0.0005} \\
0.00867_{-0.00031}^{+0.00029} & 0.0404_{-0.0005}^{+0.0011} & 0.999146_{-0.000046}^{+0.000021}\end{array}\right)$ \\
\hline \multicolumn{3}{|c|}{ Charged-lepton masses } \\
\hline $\begin{array}{l}m_{e} \\
m_{\mu} \\
m_{\tau}\end{array}$ & $\begin{array}{c}0.00061 \mathrm{GeV} \\
0.089 \mathrm{GeV} \\
1.74 \mathrm{GeV}\end{array}$ & $\begin{array}{c}0.0004866 \mathrm{GeV} \\
0.1027 \mathrm{GeV} \\
1.746 \mathrm{GeV}\end{array}$ \\
\hline \multicolumn{3}{|r|}{ Neutrino masses } \\
\hline $\begin{array}{c}m_{1} \\
m_{2} \\
m_{3} \\
\Delta m_{21}^{2} \\
\Delta m_{13}^{2}\end{array}$ & $\begin{array}{l}5.46 \cdot 10^{-2} \mathrm{eV} \\
5.53 \cdot 10^{-2} \mathrm{eV} \\
4.17 \cdot 10^{-5} \mathrm{eV} \\
7.96 \cdot 10^{-5} \mathrm{eV}^{2} \\
2.98 \cdot 10^{-3} \mathrm{eV}^{2}\end{array}$ & $\begin{array}{c}- \\
- \\
- \\
(7.50 \pm 0.185) \cdot 10^{-5} \mathrm{eV}^{2} \\
\left(2.47_{-0.067}^{+0.069}\right) \cdot 10^{-3} \mathrm{eV}^{2}\end{array}$ \\
\hline \multicolumn{3}{|r|}{ Lepton mixing matrix } \\
\hline $\begin{array}{c}\left|U_{\mathrm{PMNS}}\right| \\
\left\langle m_{\beta \beta}\right\rangle \\
\mathrm{J} \\
\theta_{12} \\
\theta_{23} \\
\theta_{13}\end{array}$ & $\begin{array}{c}\left(\begin{array}{ccc}0.76 & 0.63 & 0.13 \\
0.39 & 0.58 & 0.72 \\
0.52 & 0.52 & 0.68\end{array}\right) \\
0.013 \mathrm{eV} \\
0.019 \\
39.7^{\circ} \\
46.5^{\circ} \\
7.2^{\circ}\end{array}$ & 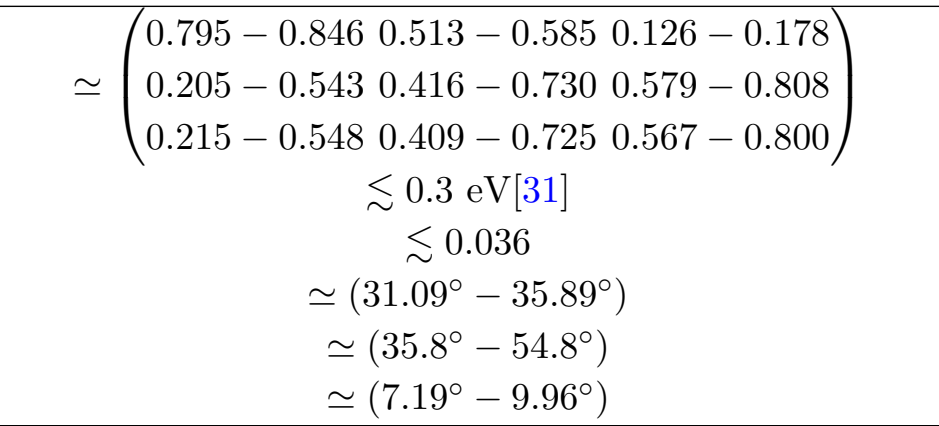 \\
\hline
\end{tabular}

Table 4. Fitted versus experimental values of the mass parameters. If other is not explicitly stated, we use the values from [28] for CKM, ref. [29] for running charged-fermion masses at $M_{Z}$ and ref. [30] for neutrino parameters. For the inverted hierarchy scenario, which is relevant for our model, $0.013 \mathrm{eV} \lesssim\left\langle m_{\beta \beta}\right\rangle \lesssim 0.05 \mathrm{eV}$. Experimental values of charged-lepton masses are known to a better precision than quoted.

on approximate wave functions, for this study, we performed a detailed calculation of the overlaps, starting from our benchmark parameters and numerical solutions for the spherical 
compactification. The strongest constraint still comes from the $K_{L}^{0} \rightarrow \bar{\mu} e($ or $\rightarrow \mu \bar{e})$ decay and reads

$$
1 / R \gtrsim 50 \mathrm{TeV}
$$

This is the constraint we used when tuning the scalar sector. The prediction of any model with slightly broken family symmetry would be the observation of $K \rightarrow \bar{\mu} e$ decay as well as $B^{0} \rightarrow K^{0} \bar{\mu}$ e (or their charge conjugate) decay [33] at a level less suppressed than either $\mu \rightarrow e \gamma$ or $\mu \rightarrow e \bar{e} e$, or $\mu-e$ conversion on nuclei (the latter FCNC processes indeed violate family number).

As pointed out before [16], the models we are considering here may be generic of similar ones with more general geometry (or simply a yet unexplored range of parameters) ${ }^{3}$. In fact, the above constraint on the KK scale rests entirely on the value of the overlaps $\varkappa$. Models or fits providing lower $\varkappa$ values would allow to reduce the mass of the $Z^{\prime}$ and other recurrences, and maybe bring them in the reach of accelerator searches. Such an analysis can be conducted in a fairly model-independent way, since it directly relates constraints from precision data to LHC searches. For this reason, a search for possible signals is certainly in order already at LHC. Of course, the price to pay for a low $\varkappa$ is also a reduced production of the recurrences. The relevant processes are the production of $\gamma^{\prime}, Z^{\prime}$ or $W^{\prime}$ bosons (the actual KK mode) which carry a nonzero winding number and thus decays with family violation. The signal is indeed quite striking $\left(\mu^{+} e^{-}\right.$pairs exceeding $\mu^{-} e^{+}$pairs, for instance).

Here we ignore the KK recurrences of the gluons. They are of course easy to include, but the corresponding signals are difficult to disentangle (as they need precise quark identification) while enjoying larger production rates. Another point is that we refer to $Z^{\prime}$, $\gamma^{\prime}$, etc.; it should be noted that, in first approximation (ignoring the scale of EW symmetry breaking as compared to the compactification scale), those modes are degenerate and should thus be added coherently. It has been argued (see ref. [34]) that loop corrections might instead lift the degeneracy into a $W_{3}^{\prime}-B^{\prime}$ mode rather than $Z^{\prime}-\gamma^{\prime}$ way. We leave this question open, as we are rather wary of getting into (non-renormalizable) loop calculations.

We have checked that the couplings of the scalar boson $H$ to vector bosons and fermions are very similar to the SM, and therefore we do not predict any particular signature here. This is true for tree-level couplings, but for loop-induced couplings as well (like $\gamma \gamma$ or gluon-gluon), to which the KK contributions appear to be negligible. Note that the bound (3.4) is much stronger than the ones usually quoted to satisfy other precision tests, like the $S$ and $T$ parameters (even if the context is somewhat different here) [35] and that they arise at the (safer from our point of view) tree level, instead as through loops like e.g. in Ref. [36].

\footnotetext{
${ }^{3}$ In the framework chosen in the present paper, we would need to reduce strongly $1 / R$ and the overlaps $\varkappa$ accordingly. We have found that this possibility was strongly restricted, in particular by the smallness of the Standard Model Scalar mass $(125 \mathrm{GeV})$. Alternatives would at least imply modifications in the scalar potential and/or in the geometry of the compactification. These considerations lie outside the scope of the present work.
} 


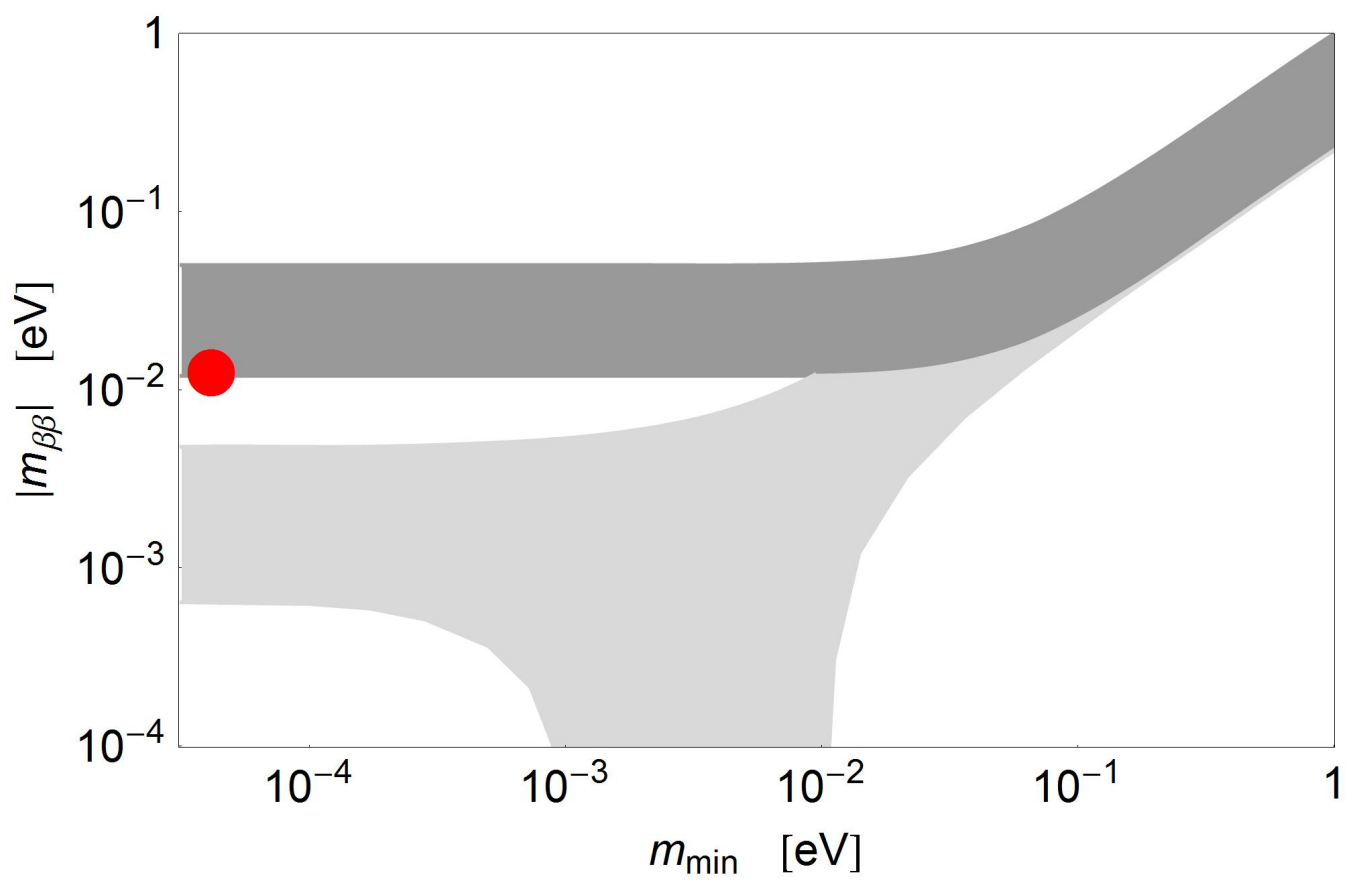

Figure 1. The effective Majorana mass $m_{\beta \beta}$ versus the minimal absolute neutrino mass. The 99\% allowed regions for the normal hierarchy (light gray) and inverse hierarchy (dark gray) [31] are shown together with the prediction of our benchmark model (the thick dot).

Still, the most striking bunch of tests (not depending on reaching the energy or sensitivity associated to the KK scale) remains in the leptonic sector, where definite predictions are done. While large mixings are a generic prediction of the approach, our benchmark fit predicts inverted hierarchy, a particular value of $C P$ violation and the smallest possible range of neutrinoless double beta decay compatible with inverted hierarchy [31, 37], cf. section 4.2 (see Figure 1). This sensitivity will only be reached by the "phase 3" of the GERDA experiment [38].

A falsifying test for the present version of the model would be a discovery of the fourth active neutrino. While the model is capable of accommodating more than three families, its nice feature of the natural explanation of large neutrino mixings within the same mechanism which provides the quark mass hierarchy would fail for even number of families. The case of five generations is beyond the scope of the present work.

\section{Conclusions}

To summarize, we review and update the six-dimensional model of flavour which gives a natural and simultaneous explanation of two very different mass-matrix hierarchies: one for charged fermions and another one for neutrinos. Both hierarchies are governed by the same small parameter. We formulate a benchmark model which gives a satisfactory fit of all masses and mixings, given the latest measurements of the scalar-boson mass and of neutrino mixing angles and taking advantage of new, more precise, numerical solutions 
of the field equations. We give distinctive experimental predictions which would allow to test the model in future experiments. Some alternatives are still possible. For instance, the charge assignments in table 1 could be modified, which could change the perceived hierarchy of operators while the full parameter space has of course not been explored.

\section{Acknowledgments}

We are indebted to D. Gorbunov, E. Nugaev and M. Tytgat for helpful discussions. M.L. and S.T. thank the Service de Physique Théorique at Université Libre de Bruxelles for kind hospitality. This work is funded in part by IISN and by Belgian Science Policy (IAP VII/37). This work was supported in part by the grant of the President of the Russian Federation NS-5590.2012.2 (M.L. and S.T.); by the Russian Foundation for Basic Research (grants 12-02-00653 (M.L.); 11-02-01528, 12-02-01203, 13-02-01311 and 13-0201293 (S.T.)); by the RF Ministry of Science and Education (agreements 8142 (S.T.) and 14.B37.21.0457 (M.L. and S.T.)) and by the "Dynasty" foundation (M.L.).

\section{References}

[1] ATLAS Collaboration, G. Aad et al., Observation of a new particle in the search for the Standard Model Higgs boson with the ATLAS detector at the LHC, Phys.Lett. B716 (2012) 1-29, [arXiv: 1207.7214].

[2] CMS Collaboration, S. Chatrchyan et al., Observation of a new boson at a mass of $125 \mathrm{GeV}$ with the CMS experiment at the LHC, Phys.Lett. B716 (2012) 30-61, [arXiv:1207.7235].

[3] ATLAS Collaboration, B. Mansoulie et al., "Combination of results on the BEH Boson in ATLAS." Talk at the "XLVIIIth Rencontres de Moriond session devoted to Electroweak interactions and unified theories", La Thuile, March 2-9, 2013.

[4] CMS Collaboration, M. Chen et al., "Combination and interpretation of Scalar Boson search results from CMS." Talk at the "XLVIIIth Rencontres de Moriond session devoted to Electroweak interactions and unified theories", La Thuile, March 2-9, 2013.

[5] F. Englert and R. Brout, Broken Symmetry and the Mass of Gauge Vector Mesons, Phys.Rev.Lett. 13 (1964) 321-323.

[6] P. W. Higgs, Broken Symmetries and the Masses of Gauge Bosons, Phys.Rev.Lett. 13 (1964) 508-509.

[7] T2K Collaboration, K. Abe et al., Indication of Electron Neutrino Appearance from an Accelerator-produced Off-axis Muon Neutrino Beam, Phys.Rev.Lett. 107 (2011) 041801, [arXiv: 1106. 2822].

[8] DAYA-BAY Collaboration, F. An et al., Observation of electron-antineutrino disappearance at Daya Bay, Phys.Rev.Lett. 108 (2012) 171803, [arXiv:1203.1669].

[9] M. Libanov and S. V. Troitsky, Three fermionic generations on a topological defect in extra dimensions, Nucl.Phys. B599 (2001) 319-333, [hep-ph/0011095].

[10] J. Frere, M. Libanov, and S. V. Troitsky, Three generations on a local vortex in extra dimensions, Phys.Lett. B512 (2001) 169-173, [hep-ph/0012306]. 
[11] J. Frere, M. Libanov, and S. V. Troitsky, Neutrino masses with a single generation in the bulk, JHEP 0111 (2001) 025, [hep-ph/0110045].

[12] M. V. Libanov and E. Y. Nougaev, Towards the realistic fermion masses with a single family in extra dimensions, JHEP 0204 (2002) 055, [hep-ph/0201162].

[13] M. Libanov and E. Y. Nugaev, Hierarchical fermionic mass pattern and large extra dimensions, Surveys High Energ.Phys. 17 (2002) 165-171.

[14] J. Frere, M. Libanov, E. Nugaev, and S. V. Troitsky, Fermions in the vortex background on a sphere, JHEP 0306 (2003) 009, [hep-ph/0304117].

[15] J. Frere, M. Libanov, E. Nugaev, and S. V. Troitsky, Flavor violation with a single generation, JHEP 0403 (2004) 001, [hep-ph/0309014].

[16] J. Frere, M. Libanov, E. Y. Nugaev, and S. V. Troitsky, Searching for family number conserving neutral gauge bosons from extra dimensions, JETP Lett. 79 (2004) 598-601.

[17] M. Libanov and E. Y. Nugaev, Properties of the Higgs particle in a model involving a single unified fermion generation, Phys.Atom.Nucl. 70 (2007) 864-870.

[18] M. Libanov and E. Nugaev, Higgs boson with a single generation in the bulk, hep-ph/0512223.

[19] J.-M. Frere, M. Libanov, and F.-S. Ling, See-saw neutrino masses and large mixing angles in the vortex background on a sphere, JHEP 1009 (2010) 081, [arXiv: 1006.5196].

[20] M. Libanov and F. Ling, Why neutrinos are different?, PoS QFTHEP2011 (2011) 072.

[21] M. Libanov and F.-S. Ling, Flavour puzzle or Why neutrinos are different?, arXiv: 1105.6035.

[22] M. Giovannini, H. Meyer, and M. E. Shaposhnikov, Warped compactification on Abelian vortex in six-dimensions, Nucl.Phys. B619 (2001) 615-645, [hep-th/0104118].

[23] E. Witten, Superconducting Strings, Nucl.Phys. B249 (1985) 557-592.

[24] W. D. Goldberger and M. B. Wise, Renormalization group flows for brane couplings, Phys.Rev. D65 (2002) 025011, [hep-th/0104170].

[25] E. Dudas, C. Papineau, and V. Rubakov, Flowing to four dimensions, JHEP 0603 (2006) 085, [hep-th/0512276].

[26] R. Jackiw and P. Rossi, Zero Modes of the Vortex - Fermion System, Nucl.Phys. B190 (1981) 681.

[27] E. Ma and S. Pakvasa, Variation of mixing angles and masses with $Q^{2}$ in the standard six quark model, Phys.Rev. D20 (1979) 2899.

[28] Particle Data Group Collaboration, J. Beringer et al., Review of Particle Physics (RPP), Phys.Rev. D86 (2012) 010001.

[29] Z.-z. Xing, H. Zhang, and S. Zhou, Impacts of the Higgs mass on vacuum stability, running fermion masses and two-body Higgs decays, Phys.Rev. D86 (2012) 013013, [arXiv:1112.3112].

[30] M. Gonzalez-Garcia, M. Maltoni, J. Salvado, and T. Schwetz, Global fit to three neutrino mixing: critical look at present precision, JHEP 1212 (2012) 123, [arXiv:1209.3023].

[31] S. Bilenky and C. Giunti, Neutrinoless double-beta decay: A brief review, Mod.Phys.Lett. A27 (2012) 1230015, [arXiv: 1203.5250]. 
[32] N. Cosme, J. Frere, and L. Lopez Honorez, CP violation from dimensional reduction: Examples in (4+1)-dimensions, Phys.Rev. D68 (2003) 096001, [hep-ph/0207024].

[33] M. Libanov, N. Nemkov, E. Nugaev, and I. Timiryasov, Heavy-meson physics and flavour violation with a single generation, JHEP 1208 (2012) 136, [arXiv:1207.0746].

[34] H.-C. Cheng, K. T. Matchev, and M. Schmaltz, Radiative corrections to Kaluza-Klein masses, Phys.Rev. D66 (2002) 036005, [hep-ph/0204342].

[35] M. Baak et al., Updated Status of the Global Electroweak Fit and Constraints on New Physics, Eur.Phys.J. C72 (2012) 2003, [arXiv:1107.0975].

[36] U. Haisch and A. Weiler, Bound on minimal universal extra dimensions from $\bar{B} \rightarrow X(s) \gamma$, Phys.Rev. D76 (2007) 034014, [hep-ph/0703064].

[37] W. Rodejohann, Neutrino-less Double Beta Decay and Particle Physics, Int.J.Mod.Phys. E20 (2011) 1833-1930, [arXiv:1106.1334].

[38] GERDA Collaboration, K. Ackermann et al., The GERDA experiment for the search of $0 \nu \beta \beta$ decay in ${ }^{76} G e$, Eur.Phys.J. C73 (2013) 2330, [arXiv:1212.4067]. 